\title{
Malaria Surveillance of Entry People During the COVID-19 Epidemic - Guangdong Province, China, October 2020-May 2021
}

\author{
De Wu ${ }^{1}$; Zhuohui Deng ${ }^{1}$; Rongxin Lin ${ }^{1}$; Qiang $\mathrm{Mao}^{1}$; Wenchen Lu' ${ }^{1}$; \\ Caiwen Ruan ${ }^{1}$; Yongzhen Cen ${ }^{1}$; Ning $\mathrm{Xiao}^{2}$; Tie Song ${ }^{1, *}$
}

\section{Summary \\ What is already known about this topic? \\ Malaria control was affected by the coronavirus disease 2019 (COVID-19) pandemic. This study conducted active case finding for key flights and key populations to determine malaria transmission. \\ What is added by this report? \\ Surveillance for malaria was conducted for entry personnel coming from areas affected by malaria. It is estimated that at least 100,000 tests were conducted in Guangdong Province; 154 cases were confirmed during the surveillance. \\ What are the implications for public health practice? \\ To maintain the malaria elimination status, comprehensively maintaining a sensitive and effective surveillance response system is especially important.}

Malaria is a parasitic infection transmitted by Anopheles mosquitos that is a major threat to global health. There were an estimated 229,000 cases and 409,000 deaths globally in 2019 (1). Historically, Guangdong is one of the most affected provinces in China by the threat of malaria. The average annual incidence rate in the whole province was between $1.22-183.23$ per 10,000 from 1950-1980 (2), and the most historically dominant strains were Plasmodium falciparum and P. vivax in Guangdong. After more than half a century of effort to control the spread of malaria, indigenous cases had been completely eliminated in Guangdong Province since 2010 (3). Since then, as malaria is one of the main imported vector-borne diseases in Guangdong Province, the main goal of malaria prevention and control is to prevent local transmission caused by imported cases. From 2011 to 2019, the average number of imported malaria cases ranged from 136-206 per year (4). Thus the threat of imported cases cannot be ignored. However, since the coronavirus disease 2019
(COVID-19) pandemic emerged in December of 2019 (5), the early diagnosis and control of malaria has been difficult because it has similar clinical symptoms to COVID-19 in the early stages of the disease. For this reason, we carried out malaria detection and surveillance research in COVID-19 quarantine sites and other key places.

In order to strengthen the surveillance of malaria at COVID-19 quarantine sites and entry ports, to detect malaria cases early, and ultimately achieve the goal of accurately blocking the spread of malaria, we have required the following 3 categories of entry personnel from malaria-endemic areas to be screened for malaria since the resumption of international flights in October 2020: 1) people who have symptoms, including fever, headache, chills, etc.; 2) people who have accompanied confirmed malaria cases, such as those who work and live together abroad; and 3) people on flights with more than one confirmed malaria case. The third category was encouraged to be expanded in scope in qualified areas to include all inbound personnel in highly affected endemic areas. People grouped in any of the 3 categories would undergo the first screening test on the first day of entering China and undergo the second test on the day before terminating their quarantine period, i.e., the 14 th day of quarantine. The samples were collected via peripheral blood through a finger prick, and the detection reagent was Plasmodium antigen rapid detection test (RDT). If the test was positive, the patient would be sent to designated hospitals to collect $3 \mathrm{~mL}$ of venous blood, and further microscopic examination was conducted in the hospital. Samples with positive microscopic examinations were sent to the responsible CDC for microscopic and nucleic acid re-examination and species identification.

To effectively control the spread of malaria, epidemic point disposal and vector control have been strengthened further. Response measures were implemented for quarantine sites, designated hospitals, 
and their surrounding areas and included surveillance of Anopheles vectors and mosquito vector density and control of suspected breeding places. Anti-mosquito isolation was carried out for suspected screening person No Anopheles vectors were found, and no secondary cases were detected in the isolated areas where cases occurred.

From October 2020 to May 2021, it was estimated that at least 100,000 tests were conducted in Guangdong Province; 154 cases were confirmed, of which 3 cases were also infected with coronavirus disease 2019 (COVID-19). A total of 151 samples (excluding 3 cases of COVID-19 coinfection) were sent to Guangdong CDC. The time from case discovery to diagnosis and species identification was completed within 3 days, and the samples were sent to Guangdong CDC for further detection and analysis within 5 days. The results of species identification showed that falciparum malaria was the most common $(87.7 \% ; 135 / 154$ of cases), followed by ovale malaria $(7.1 \% ; 13 / 154)$, vivax malaria $(3.9 \% ; 6 / 154)$, malariae malaria $(0.6 \% ; 1 / 154)$, and mixed infection $(0.6 \%$; 1/154) (Table 1).

All cases originated from at least 23 distinct African countries, of which the Democratic Republic of Congo, Cameroon, Nigeria, the Republic of Congo, and Uganda were the top 5 countries with the largest number of cases, with a combined total of 95 cases, accounting for $61.7 \%$ of all cases. According to the registered residence of the infected patients, the imported cases were residents of 22 provincial-level administrative divisions (PLADs). Among them, Henan, Guangdong, Shandong, Hubei, and Hunan were the 5 provinces of residence with the most cases, accounting for $51.3 \%$ of all cases. All imported cases were concentrated in the COVID-19 quarantine sites of 8 cities, of which Guangzhou had the most cases with $120(77.9 \%)$, followed by Foshan and Shenzhen (Figure 1). The main occupation types of infected patients were workers and migrant workers; the age distribution was mainly concentrated in adults aged 20-60 years. In addition, these imported cases were found through active case finding: 3 cases were found at the port of entry, 5 cases were found by close contacts, and the other cases were found in COVID19 quarantine sites.

\section{DISCUSSION}

After 2017, China has achieved the goal of zero local cases (๑), and imported malaria has become the main target of malaria control in China. From 2017 to 2019, 8,202 malaria cases were imported into China, including 610 cases in Guangdong, accounting for $7.4 \%$ of all cases $(4,6)$. Therefore, Guangdong Province is one of the main malaria import provinces in China. According to surveillance data in 2002, $A n$. anthropophagus, An. sinensis, An. minimus, and $A n$. riyuetan were confirmed as natural vectors of malaria transmission in Guangdong province, of which $A n$. anthropophagus and An. minimus were the highest contributing vectors of the malaria epidemic in Guangdong Province, followed by An. sinensis ( 7 ). Due to the high annual average temperature, heavy rainfall, and long annual average activity time of vectors in Guangdong Province, the risk of local transmission or secondary cases caused by imported malaria is high. Since most of the prevention and control resources have been applied to COVID-19 prevention and control work, other infectious diseases were overlooked. For this reason, Guangdong Province began screening blood samples from people returning

TABLE 1. Distribution of Plasmodium types in Guangdong Province from October 2020 to May 2021.

\begin{tabular}{|c|c|c|c|c|c|c|c|c|c|c|c|c|}
\hline \multirow{2}{*}{ Time } & \multicolumn{2}{|c|}{ P. vivax } & \multicolumn{2}{|c|}{ P. falciparum } & \multicolumn{2}{|c|}{ P. malariae } & \multicolumn{2}{|c|}{ P. ovael } & \multicolumn{2}{|c|}{ Mixed infection } & \multicolumn{2}{|c|}{ Total } \\
\hline & Cases & Death & Cases & Death & Cases & Death & Cases & Death & Cases & Death & Cases & Death \\
\hline October, 2020 & 0 & 0 & 19 & 1 & 0 & 0 & 1 & 0 & 0 & 0 & 20 & 1 \\
\hline November, 2020 & 1 & 0 & 21 & 0 & 0 & 0 & 2 & 0 & 0 & 0 & 24 & 0 \\
\hline December, 2020 & 3 & 0 & 20 & 0 & 1 & 0 & 0 & 0 & 0 & 0 & 24 & 0 \\
\hline January, 2021 & 0 & 0 & 16 & 0 & 0 & 0 & 3 & 0 & 0 & 0 & 19 & 0 \\
\hline February, 2021 & 1 & 0 & 13 & 0 & 0 & 0 & 1 & 0 & 0 & 0 & 15 & 0 \\
\hline March, 2021 & 0 & 0 & 15 & 0 & 0 & 0 & 0 & 0 & 0 & 0 & 15 & 0 \\
\hline April, 2021 & 0 & 0 & 16 & 0 & 0 & 0 & 3 & 0 & 1 & 0 & 20 & 0 \\
\hline May, 2021 & 1 & 0 & 15 & 0 & 0 & 0 & 1 & 0 & 0 & 0 & 17 & 0 \\
\hline Total & 6 & 0 & 135 & 1 & 1 & 0 & 11 & 0 & 1 & 0 & 154 & 1 \\
\hline
\end{tabular}




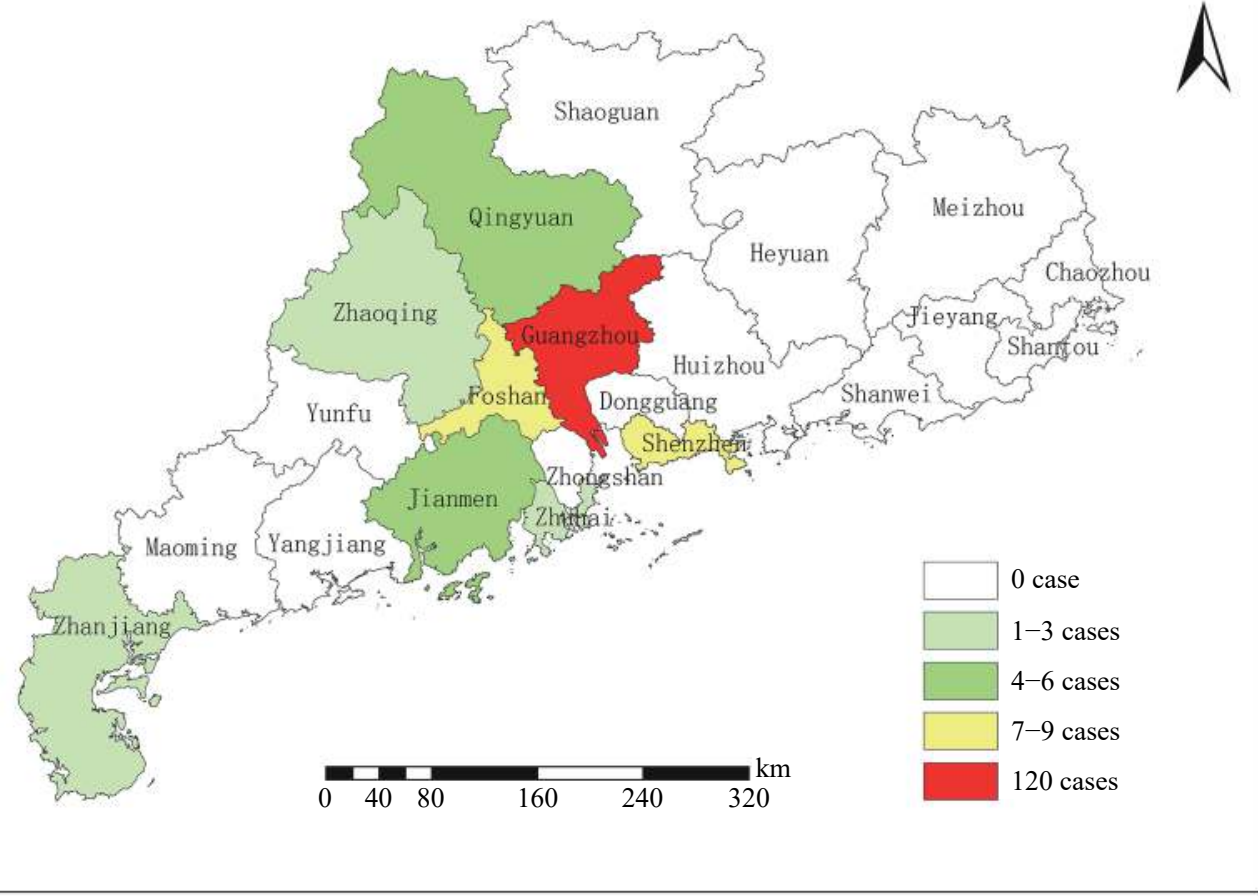

FIGURE 1. Distribution of reported malaria cases in Guangdong Province from October 2020 to May 2021.

from malaria-endemic areas such as Africa and Southeast Asia for RDT screening at all COVID-19 quarantine sites. Following the discovery of cases, the target population has gradually expanded to include travel companions and people on the same flight as infected patients. The number of screenings has been increased from one to two, greatly increasing the rate of early detection of cases.

The limitations of this study mainly include the following two points. First, the monitoring time is short, which can not fully reflect the epidemic characteristics of imported malaria cases. Second, there is a lack of mosquito vector monitoring data at quarantine points, which can not well analyze the transmission risk after malaria input.

Falciparum malaria has the highest fatality rate and is the main species of malaria imported into China. Among Chinese travellers, the mortality rate of Plasmodium falciparum infection was between 1\%-2\% (8). In this active monitoring process, 135 cases of falciparum malaria were found. Due to timely detection and treatment, the case-fatality rate was less than $1 \%$. In addition, we have successfully diagnosed 3 cases of COVID-19 complicated with Plasmodium infection. Because COVID-19 is caused by a highly pathogenic microorganism (9), biosafety lab 3 (BSL-3) personal protection is necessary for the process of malaria screening and testing. Through the effective development of rapid screening and confirmation of malaria in COVID-19 quarantine sites, so far, all detected malaria cases had been diagnosed and treated in time, and the malaria epidemic situation has been handled quickly and effectively. No second-generation cases caused by imported malaria and local epidemic spread occurred. The results showed that the measures to prevent imported and re-transmitted malaria were powerful and effective in Guangdong Province during the COVID-19 pandemic.

Acknowledgments: Participating staff members at the 21 CDCs.

\section{doi: $10.46234 / \mathrm{ccdcw} 2021.180$}

\# Corresponding author: Tie Song, tsong@cdcp.org.cn.

\footnotetext{
${ }^{1}$ Institute for Parasitic Disease Control and Prevention, Center for Disease Control and Prevention of Guangdong, Guangzhou, Guangdong, China; ${ }^{2}$ National Institute of Parasitic Diseases, Chinese Center for Disease Control and Prevention, Shanghai, China.
}

Submitted: July 06, 2021; Accepted: August 24, 2021

\section{REFERENCES}

1. World Health Organization: WHO Guidelines for malaria. https:// www.ncbi.nlm.nih.gov/books/NBK568497/. [2021-8-20].

2. Pan B, Ruan CW, Lin RX, Pei FQ, Zhang QM. Epidemiological analysis of malaria epidemic situation in Guangdong Province. Chin J 
Schistosomiasis Control 2013;25(1):40 - 3. http://dx.doi.org/10.3969/ j.issn.1005-6661.2013.01.010. (In Chinese).

3. Lin RX, Pan B, Deng ZH, Ruan CW, Pei FQ. Mid-term assessment report of Malaria Elimination Action Plan in Guangdong. Chin Trop Med 2016;16(4):349 - 53. http://dx.doi.org/10.13604/j.cnki.46-1064/ r.2016.04.11. (In Chinese).

4. Chen JD, Lin RX, Deng ZH, Pan B, Pei FQ, Lu WC, et al. Analysis on the epidemic situation of imported malaria in Guangdong Province from 2011 to 2019. J Trop Dis Parasitol 2020;18(4):197 - 201. http://dx. doi.org/10.3969/j.issn.1672-2302.2020.04.002.

5. Zhu N, Zhang DY, Wang WL, Li XW, Yang B, Song JD, et al. A novel coronavirus from patients with pneumonia in China, 2019. N Engl J Med 2020;382(8):727 - 33. http://dx.doi.org/10.1056/NEJMoa2001 017.

6. Hu XF, Wu S, Weng YQ, Han H, Tian LL, Lü YS, et al. Global malaria epidemic and countermeasures for imported cases in mainland China.
Disease Surveillance. 1-8. http://kns.cnki.net/kcms/detail/11.2928.R 20210513.1402.002.html. [2021-08-20]. (In Chinese).

7. Pan B, Zhu TH, Zang QM, Lin RX, Wu XG. Investigation on distribution, ecological feature and malaria transmission effect of anopheles in Guangdong province. Chin J Parasit Dis Control 2002;15(5):257 - 60. http://dx.doi.org/10.3969/j.issn.1673-5234.2002. 05.001. (In Chinese).

8. Lai SJ, Wardrop NA, Huang ZJ, Bosco C, Sun JL, Bird T, et al. Plasmodium falciparum malaria importation from Africa to China and its mortality: an analysis of driving factors. Sci Rep 2016;6:39524. http://dx.doi.org/10.1038/srep39524.

9. Li XC, Xu SY, Yu MQ, Wang K, Tao Y, Zhou Y, et al. Risk factors for severity and mortality in adult COVID-19 inpatients in Wuhan. J Allergy Clin Immunol 2020;146(1):110 - 8. http://dx.doi.org/10. 1016/j.jaci.2020.04.006. 